\title{
Article
}

\section{Transcriptome Analysis in Vulvar Squamous Cell Cancer}

\author{
Katharina Prieske 1,2,3,*, Malik Alawi ${ }^{4} \mathbb{D}$, Anna Jaeger ${ }^{1}$, Maximilian Christian Wankner ${ }^{5}$, Kathrin Eylmann ${ }^{1}$, \\ Susanne Reuter ${ }^{1}$, Patrick Lebok ${ }^{6}$, Eike Burandt ${ }^{6}{ }^{(D}$, Niclas C. Blessin ${ }^{6}$, Barbara Schmalfeldt ${ }^{1}$, \\ Leticia Oliveira-Ferrer ${ }^{1}$, Simon A. Joosse ${ }^{2,5,+}$ (D) and Linn Woelber $1,3,+$ (D)
}

1 Department of Gynecology and Gynecologic Oncology, University Medical Center Hamburg-Eppendorf, 20246 Hamburg, Germany; a.jaeger@uke.de (A.J.); k.eylmann@uke.de (K.E.); s.reuter@uke.de (S.R.); b.schmalfeldt@uke.de (B.S.); ferrer@uke.de (L.O.-F.); lwoelber@uke.de (L.W.)

2 Mildred Scheel Cancer Career Center HaTriCS4, University Medical Center Hamburg-Eppendorf, 20246 Hamburg, Germany; s.joosse@uke.de

3 Colposcopy Clinic at the Jerusalem Hospital Hamburg, 20357 Hamburg, Germany

4 Bioinformatics Core, University Medical Center Hamburg-Eppendorf, 20246 Hamburg, Germany; m.alawi@uke.de

5 Department of Tumor Biology, University Medical Center Hamburg-Eppendorf, 20246 Hamburg, Germany; maximilian.wankner@semmelweis-hamburg.de

6 Institute of Pathology, University Medical Center Hamburg-Eppendorf, 20246 Hamburg, Germany; p.lebok@uke.de (P.L.); e.burandt@uke.de (E.B.); n.blessin@uke.de (N.C.B.)

* Correspondence: k.prieske@uke.de

+ Authors contributed equally.

check for updates

Citation: Prieske, K.; Alawi, M.; Jaeger, A.; Wankner, M.C.; Eylmann, K.; Reuter, S.; Lebok, P.; Burandt, E.; Blessin, N.C.; Schmalfeldt, B.; et al. Transcriptome Analysis in Vulvar Squamous Cell Cancer. Cancers 2021, 13, 6372. https://doi.org/10.3390/ cancers 13246372

Academic Editors: Mario Preti and Denis Querleu

Received: 14 November 2021 Accepted: 16 December 2021 Published: 19 December 2021

Publisher's Note: MDPI stays neutral with regard to jurisdictional claims in published maps and institutional affiliations.

Copyright: (C) 2021 by the authors. Licensee MDPI, Basel, Switzerland. This article is an open access article distributed under the terms and conditions of the Creative Commons Attribution (CC BY) license (https:/ / creativecommons.org/licenses/by/ $4.0 /)$.
Simple Summary: The number of women, especially younger women, diagnosed with vulvar cancer, has been rising mainly due to the infection with human papilloma virus (HPV) over the last years. In contrast to other tumor entities, limited information on the underlying genetic changes is available, and thus treatment advances, especially the development of personalized treatments, are hampered. We aimed to explore the RNA expression profiles in a group of 24 vulvar cancer samples in order to detect potential prognostic markers and therapeutic targets in order to establish to a more profound understanding of vulvar cancer carcinogenesis.

Abstract: To date, therapeutic strategies in vulvar squamous cell carcinoma (VSCC) are lacking molecular pathological information and targeted therapy hasn't been approved in the treatment of VSCC, yet. Two etiological pathways are widely accepted: HPV induced vs. HPV independent, associated with chronic skin disease, often harboring TP53 mutations (mut). The aim of this analysis was to analyze the RNA expression patterns for subtype stratification on VSCC samples that can be integrated into the previously performed whole exome sequencing data for the detection of prognostic markers and potential therapeutic targets. We performed multiplex gene expression analysis (NanoString) with 770 genes in 24 prior next generation sequenced samples. An integrative data analysis was performed. Here, 98 genes were differentially expressed in TP53mut vs. HPV+ VSCC, in the TP53mut cohort, where 56 genes were upregulated and 42 were downregulated in comparison to the HPV+ tumors. Aberrant expression was primarily observed in cell cycle regulation, especially in HPV+ disease. Within the TP53mut group, a distinct cluster was identified that was correlated to a significantly worse overall survival $(p=0.017)$. The RNA expression profiles showed distinct patterns with regard to the known VSCC subtypes and could potentially enable further subclassification in the TP53mut groups

Keywords: vulvar cancer 1; sequencing 2; transcriptome 3; TP53 4; HPV 5; RNA 6

\section{Introduction}

Over the last decades, the incidence of vulvar squamous cell carcinoma (VSCC) has continuously been rising [1-3]. Two etiological pathways are widely accepted [4,5]. Approximately $40 \%$ of VSCCs are attributable to high-risk human papillomavirus (HPV), occurring 
more frequently in younger women [6]. The majority of VSCCs develop in postmenopausal women, are HPV independent, and are initiated by TP53 mutations. These tumors develop from chronic dystrophic skin disease, mostly lichen sclerosus as a facultative precancerous condition. A third pathway causing VSCC, which arises independently of HPV infection or TP53 mutations, has been proposed but is currently poorly understood $[7,8]$. While primary treatment comprises surgery with radical local excision of the primary tumor and surgical staging of the groins with or without adjuvant chemoradiation in case of advanced nodal involvement [9], therapeutic options are extremely limited in the case of surgically nonresectable recurrent VSCC or occurrence of distant metastases [10]. Treatment strategies, such as antiangiogenetic treatment or immunecheckpoint inhibition, are then generally experimental and adapted from cervical, anal, or head and neck cancer (HNSCC) [11-13]. Thorough molecular characterization, as performed by The Cancer Genome Atlas (TCGA) research network, with large scale genomic, epigenomic, transcriptomic, and proteomic analyses has deepened our understanding of various types of human cancers and has led to more accurate subtype stratification and prognoses. In many cases, potential new targets have led to investigation in clinical trials and changed the way patients are treated in the clinic. Unfortunately, vulvar cancer has not been considered by the TCGA and comparable molecular analyses are still lacking. It is intuitive that deep depth molecular characterization is a prerequisite for the characterization of potential targets in clinical trials and for improved treatment strategies in the long term.

A couple of groups have investigated the underlying genetic alterations in VSCC, mostly via next generation sequencing (NGS) panel analysis [14-17], two groups including our own, applied whole exome sequencing (WES) [5,18]. Due to huge differences in methodology, HPV detection, and sample size, the comparison was hampered. However, beyond TP53 as a key oncogenic driver, alterations of the prooncogenic PI3K/AKT/mTOR pathway, including HRAS, KRAS, PIK3CA, KMT2D, and PTEN mutations, have consistently been reported across different studies. In addition, mutations in potential regulators of the PI3K/Akt/mTOR pathway like FBXW7, NBPF1, and TSC2 were found, as well as in WES and hotspot analyses $[5,8,18]$. Han et al. reported PI3KCA mutations, but also high numbers of copy number gains, leading to a PIK3CA alteration rate of $60 \%$ in their HPV positive cohort. Recently, methylation profiling was performed in a set of 18 primary VSCC, including 3 $\mathrm{HPV}+$ samples and compared to normal tissue [19]. Most of the genes that were found to be hypermethylated in VSCCs (e.g., ZSCAN1, ZNF135, ZNF471, or TBX3) are involved in transcription regulator activity.

While DNA sequencing can identify important drivers for malignant transformation of the cell, it insufficiently describes its actual phenotype. Transcriptome profiling has led to a more profound molecular understanding of carcinogenesis, expanding on the knowledge of distinct prognostic relevant subtypes, biomarker development, and the impact of the tumor microenvironment $[20,21]$.

So far, very few groups have performed RNA expression analysis in VSCC. In a small set of five VSCC samples with matched normal controls, aberrant regulation of cell cycle, growth, and proliferation; cell death; and cellular development were detected. Recently, another transcriptome analysis of 23 VSCC and one control vulvar tissue was performed. Two distinct groups, defined by HPV status, with several differentially expressed genes (e.g., STMN1, FOXO6, FCGBP DMBX1, and GBX1) were detected. In the pathway analysis, enrichment for cytokine receptor and inflammatory signaling was revealed in the HPV+ tumors [22,23].

The aim of this analysis is to combine genomic and transcriptomic information in order to identify prognostic markers, deregulated pathways, and potential targets for new treatment strategies in VSCC. Therefore, 24 of the previously sequenced samples were subjected to multiplex gene expression analysis, including 770 genes. 


\section{Materials and Methods}

\subsection{Patients and Tumor Tissue}

The tissue of 24 fresh frozen VSCC samples that had previously been subjected to WES were available [5]. Somatic variants from our WES analysis in vulvar cancer are provided in Table S1. Tissue samples were obtained at the University Medical Center Hamburg-Eppendorf during surgeries performed between 1998 and 2011. All samples were immediately snap frozen and stored in liquid nitrogen at $-196^{\circ} \mathrm{C}$. Every sample was assessed on cryo-cut sections stained with hematoxylin and eosin. If necessary, the stromal parts were removed by scraping them using a scalpel to obtain at least $60 \%$ tumor cells in the sample used for RNA extraction. Pathological studies were carried out by Dr. Lebok, who is a specialized gynecopathologist. All of the patients enrolled gave written informed consent to access their tissue and review their clinical records, according to our investigational review board and ethics committee guidelines. (Ethics Committee of the Medical Board Hamburg reference number 190504). Data were retrieved from patient records and the institutional database providing information on clinicopathologic factors, and histology and therapeutic approaches. For tumor staging, the International Federation of Gynecology and Obstetrics (FIGO) stage groupings and the International Union against Cancer (UICC) tumor-node-metastasis (TNM) classification sixth edition were used for homogeneity [24,25].

\subsection{Subgroup Definition}

In our previous WES analysis of the present VSCC samples, TP53 mutation status and HPV E7 integration into the human genome were analyzed. The tumors that showed neither TP53 mutations nor HPV E7 integration were categorized as "double negatives" (OTHER).

\subsection{RNA Extraction}

Here, 20-30 cryosections (approximately $16 \mu \mathrm{m}$ ) were disintegrated using Precellys homogeniser (WVR International GmbH, Darmstadt, Germany). Subsequently, RNA was extracted using the RNAeasy Kit (Qiagen GmbH, Hilden, Germany), according to the manufacturer's instructions. RNA quantity and integrity were assessed using a Bioanalyzer device (Agilent, Santa Clara, CA, USA). Then, 200-400 ng RNA was requested for nanostring analysis, depending on the RNA quality. The range of RIN values was $>2.3-9.8$ and the bionalyzer concentration ranged from $156-1436 \mathrm{ng} / \mathrm{mL}$.

\subsection{Sequencing and Bioinformatic Analysis}

Multiplex gene expression analysis (NanoString CancerPath C2535) with 770 genes was performed in 24 of the 34 formerly sequenced VSCC samples (Table S2: Results of the differential expression analysis (TP53mut vs. HPV+). Integrative data analysis was carried out by the Bioinformatics Core of the University Medical Center Hamburg-Eppendorf.

Normalization was performed using RUVseq [26], as described previously by Bhattacharya and colleagues [27], where 38 of 40 housekeeping genes (NUBP1 and PRPF38A were excluded) and of 4 of 6 positive controls (ERCC_00035.1 and ERCC_00034.1 were excluded) were selected as a reference group for the normalization process. One dimension of unwanted variation was removed $(\mathrm{k}=1)$. Differential expression analysis was carried out with DESeq2 [27]. A gene was considered differentially expressed if the corresponding absolute $\log 2$-transformed foldchange $(\log 2 \mathrm{FC})$ was not less than 1 and, in addition, the false discovery rate (FDR) did not exceed the value of 0.1 The detection of the pathways over-represented in the set of differentially expressed genes was performed using ClusterProfiler [28], in combination with the Reactome Pathway Database [29].

\subsection{Statistical Analysis}

Statistical computations were executed using the R programming language (Foundation for Statistical Computing) and the free, online statistical tools from In-Silico Online, 
version 2.3.1 [30]. To test for associations between clinic-pathological risk factors and patient groups, cross tables were constructed and analyzed using the G-test with Williams correction. Median differences between patient groups were tested using the Wilcoxon rank test. Survival analyses were performed using Kaplan-Meier estimates accompanied with the log rank test, as well as the cox proportional hazard function for multivariable testing. Normalized gene expression counts were analyzed by unsupervised hierarchical clustering using Ward D2 linkage and Euclidian distance. For all of the statistical tests, significance was considered when $p<0.05$.

\section{Results}

\subsection{Subgroup Definition}

Among the 24 analyzed samples, four tumors were excluded from the TP53mut and HPV+ cohort and were termed "OTHER" (Table 1, for individual patient characteristics see Table S3). Two of them (24 and 47) were excluded due to TP53 wildtype (wt) status and the absence of HPV E7 integration. The two remaining samples, originally labelled as HPV+ tumors were reevaluated and subsequently excluded. In particular, sample 56 showed very few copies of E7 integration and displayed an oncogenic CCND1 mutation in WES, which is more likely to be the oncogenic driver in this tumor sample with HPV as a bystander infection. The other sample (15) was excluded, because the principal component analysis (PCA) revealed that its expression profile was more similar to that of the excluded samples 24 and 56 than to those of the other HPV positive samples. While the four aforementioned tumors $(15,24,47$, and 56) were excluded from the statistical analysis, we nonetheless included their corresponding gene expression profiles in Figure 1a.

Table 1. Patient characteristics of HPV+ and TP53mut tumors. n.a., not applicable; FD, first diagnosis; yrs, years; SNL, Sentinel (individual patient characteristics are listed in Table S3).

\begin{tabular}{|c|c|c|c|c|}
\hline Characteristics & $\begin{array}{l}\mathrm{HPV}+ \\
N=5\end{array}$ & $\begin{array}{c}\text { HPV+ } \\
\%\end{array}$ & $\begin{array}{c}\text { TP53mut } \\
N=15\end{array}$ & $\begin{array}{c}\text { TP53mut } \\
\%\end{array}$ \\
\hline $\begin{array}{l}\text { Age at FD (yrs) median } \\
\text { (range) }\end{array}$ & $\begin{array}{c}63 \\
(40-81)\end{array}$ & n.a. & $\begin{array}{c}67 \\
(38-84)\end{array}$ & n.a. \\
\hline \multicolumn{5}{|l|}{ Tumor stage } \\
\hline pT1b & 1 & 20 & 3 & 20 \\
\hline pT2 & 4 & 80 & 8 & 53.3 \\
\hline pT3 & 0 & 0 & 1 & 6.7 \\
\hline pT4 & 0 & 0 & 1 & 6.7 \\
\hline unknown & 0 & 0 & 2 & 13.3 \\
\hline \multicolumn{5}{|l|}{ Nodal status } \\
\hline unilateral groin metastases & 1 & 20 & 1 & 6.7 \\
\hline bilateral groin metastases & 0 & 0 & 1 & 6.7 \\
\hline no groin metastases & 4 & 80 & 10 & 66.7 \\
\hline unknown & 0 & 0 & 3 & 20 \\
\hline \multicolumn{5}{|l|}{ Median tumor diameter } \\
\hline $\begin{array}{c}\mathrm{mm} \\
\text { (range) }\end{array}$ & $\begin{array}{c}49.5 \\
(45-50)\end{array}$ & n.a. & $\begin{array}{c}50 \\
(15-150)\end{array}$ & n.a. \\
\hline \multicolumn{2}{|l|}{ Median depth of invasion } & \multirow[b]{2}{*}{ n.a. } & & \multirow[b]{2}{*}{ n.a. } \\
\hline $\begin{array}{c}\mathrm{mm} \\
\text { (range) }\end{array}$ & $\begin{array}{c}10 \\
(8-10)\end{array}$ & & $\begin{array}{c}7 \\
(5-28)\end{array}$ & \\
\hline esection status of vulvar prima & & & & \\
\hline
\end{tabular}


Table 1. Cont.

\begin{tabular}{|c|c|c|c|c|}
\hline Characteristics & $\begin{array}{l}\text { HPV+ } \\
N=5\end{array}$ & $\begin{array}{l}\mathrm{HPV}+ \\
\%\end{array}$ & $\begin{array}{l}\text { TP53mut } \\
N=15\end{array}$ & $\begin{array}{c}\text { TP53mut } \\
\%\end{array}$ \\
\hline R0 & 3 & 60 & 11 & 73.3 \\
\hline R1 & 1 & 20 & 2 & 13.3 \\
\hline unknown & 1 & 20 & 2 & 13.3 \\
\hline \multicolumn{5}{|l|}{ Grading } \\
\hline G1 & 1 & 20 & 1 & 6.7 \\
\hline G2 & 2 & 40 & 5 & 33.3 \\
\hline G3 & 2 & 40 & 6 & 40 \\
\hline unknown & 0 & 0 & 3 & 20 \\
\hline \multicolumn{5}{|l|}{ Vulvar surgery } \\
\hline Vulvectomy & 3 & 60 & 7 & 46.7 \\
\hline Radical local Excision & 2 & 40 & 6 & 40 \\
\hline No local surgery & 0 & 0 & 1 & 6.7 \\
\hline unknown & 0 & 0 & 1 & 6.7 \\
\hline \multicolumn{5}{|l|}{ Groin Surgery } \\
\hline $\begin{array}{l}\text { Full Groin dissection } \\
\text { (unilateral) }\end{array}$ & 1 & 20 & 2 & 13.3 \\
\hline Full Groin dissection (bilateral) & 4 & 80 & 5 & 33.3 \\
\hline SNL only (bilateral) & 0 & 0 & 3 & 20 \\
\hline $\mathrm{SLN}^{+}$followed by bilateral groin dissection & 0 & 0 & 0 & 0 \\
\hline No LNE & 0 & 0 & 4 & 26.7 \\
\hline Unknown & 0 & 0 & 1 & 6.7 \\
\hline Pelvic node dissection & 1 & 20 & 1 & 6.7 \\
\hline \multicolumn{5}{|l|}{ Adjuvant therapy } \\
\hline Radiotherapy & 2 & 40 & 3 & 20 \\
\hline Chemoradiation & 1 & 20 & 0 & 0 \\
\hline Laser & 0 & 0 & 0 & 0 \\
\hline no adjuvant therapy & 2 & 40 & 11 & 73.3 \\
\hline unknown & 0 & 0 & 1 & 6.7 \\
\hline \multicolumn{5}{|l|}{ Radiation field } \\
\hline Vulva \pm .groin \pm .pelvis & 3 & 60 & 2 & 13.3 \\
\hline Groin \pm .pelvis only & 0 & 0 & 1 & 6.7 \\
\hline unknown & 0 & 0 & 0 & 0 \\
\hline Recurrence & 1 & 20 & 3 & 20 \\
\hline \multicolumn{5}{|l|}{ Localization of recurrence (multiples possible) } \\
\hline Vulva & 1 & 20 & 3 & 20 \\
\hline Groins & 1 & 20 & 2 & 13.3 \\
\hline Recurrence free & 4 & 80 & 10 & 66.7 \\
\hline Distant metastasis in course of disease & 1 & 20 & 2 & 13.3 \\
\hline
\end{tabular}




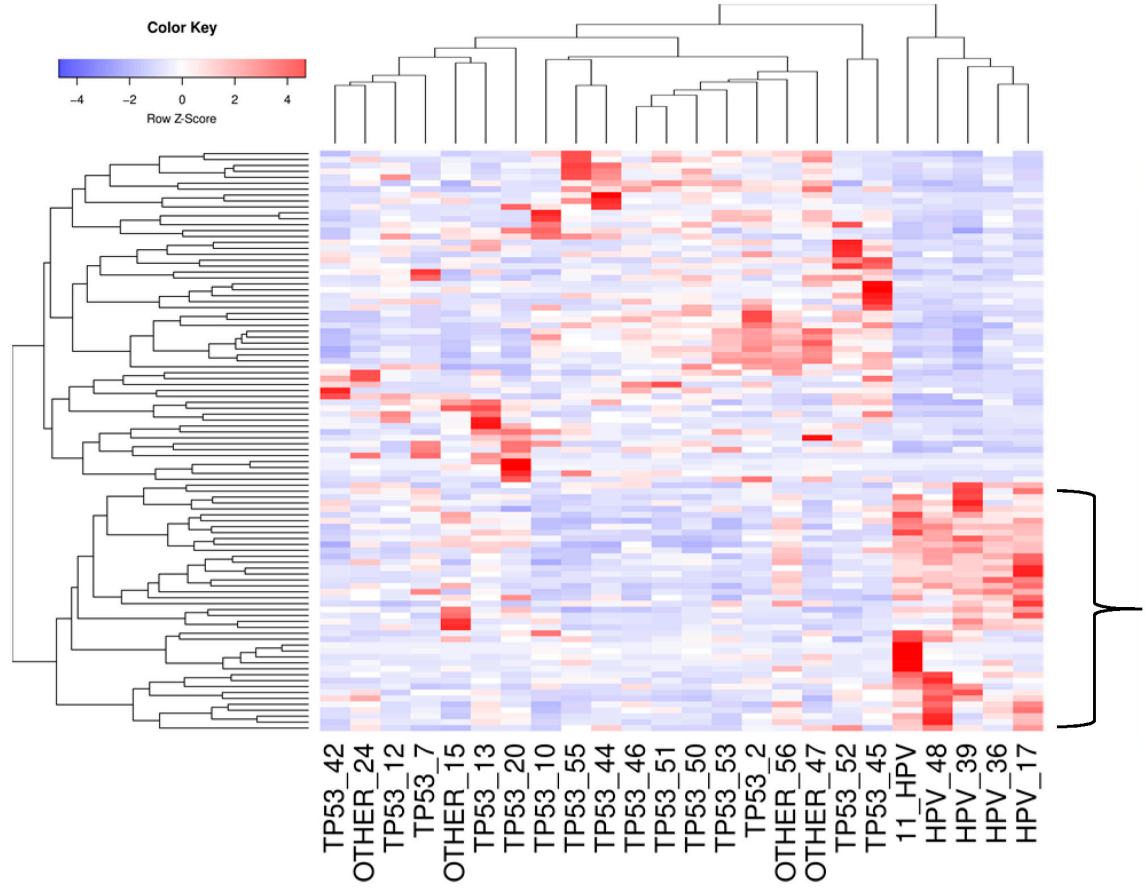

(a)

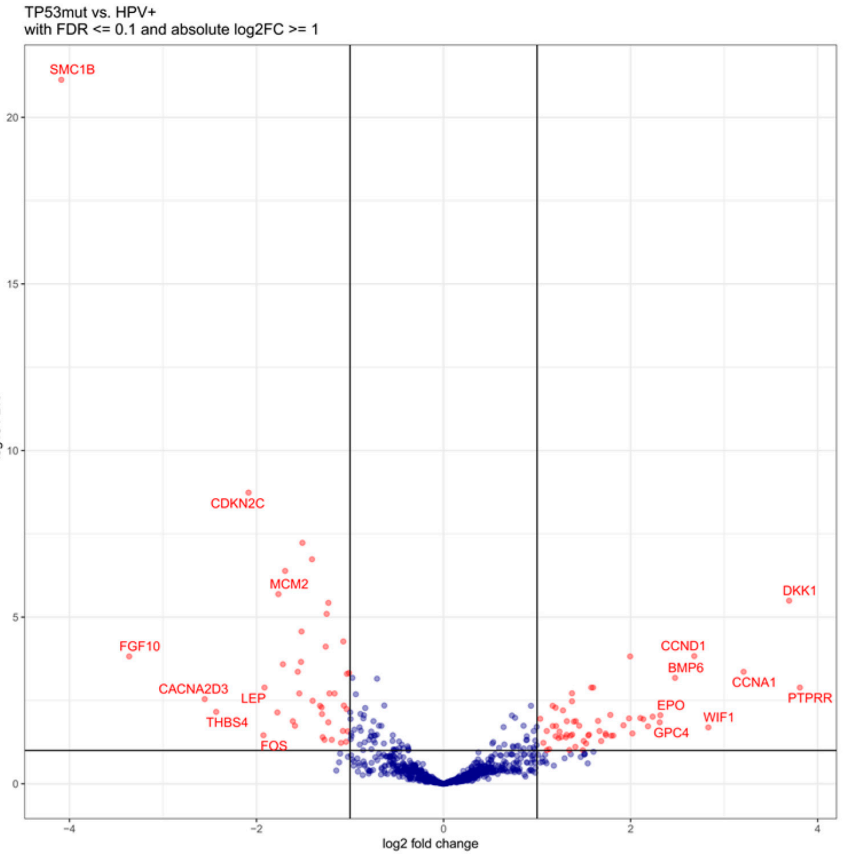

(b)

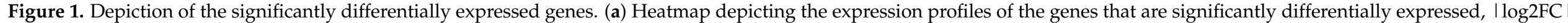

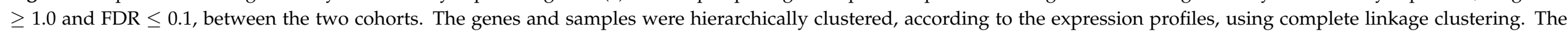

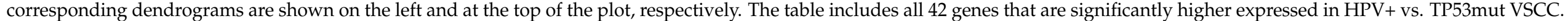

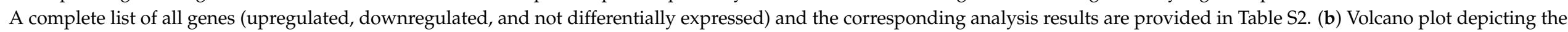

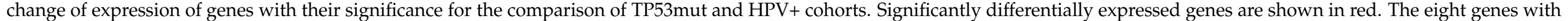
the greatest positive and the eight genes with the greatest negative $\log 2 \mathrm{FC}$ were labeled. 


\subsection{Differential Expression in Subgroups}

There were 98 genes significantly differentially expressed in primary TP53mut vs. HPV+ VSCC (Figure 1a,b). In the TP53mut cohort, 56 genes were upregulated and 42 were downregulated in comparison to HPV+ tumors (complete list of genes Table S2).

\subsubsection{TP53mut VSCC}

In the TP53mut subgroup, Dickkopf-1 (DKK-1: $\log 2 \mathrm{FC}: 3.69)$ and cell cycle regulators CCND1 (log2FC: 2.68), CCND2 (log2FC: 1.82), CCNA1 (log2FC: 3.22), and CDK6 (log2FC: 2.25) were observed to be highly upregulated compared to HPV+ VSCC. Further significantly upregulated genes in the TP53mut subgroup with a $\log 2 \mathrm{FC}$ greater than 1.25 and a normalized expression (average value of all samples) greater than 100 included LAMA1/3, WIF1, FZD3, MMP7, GRIA3, RAC2, THBS1, GPC4, IRS1, CSF3R, and CREB3L1, collagens as extracellular matrix components (COL1A1/COL1A2, COL3A1, COL5A, and COL5A2), PLCB4, FN1, INHBB, IL7R, BNIP3, and PDGFRA. See Figure $1 \mathrm{~b}$ for the genes with the highest absolute FC, independent of their base mean.

In the second step, the differential gene expression was evaluated within the TP53mut group. Using unsupervised hierarchical clustering, the TP53mut samples were subsequently separated into two groups, showing a significant differential expression of 20 genes (Figure 2a). Here, 10 genes were upregulated (TMPRSS2, IL20RA, RASAL1, MAPK8IP2, PLA2G3, PRMT8, HES5, SYK, IL1R2, and PLA2G4A) and 10 genes were downregulated (TSLP, PTPRR, IGFBP3, PRLR, ITGB3, FN1, VEGFC, DUSP10, GPC4, and RET) in group 2 in comparison to group 1 (Table 2). The highest log2FoldChange was shown in TMPRSS2, PLA2G3, IL1R2, PTPRR, TSLP, and GPC4, respectively.

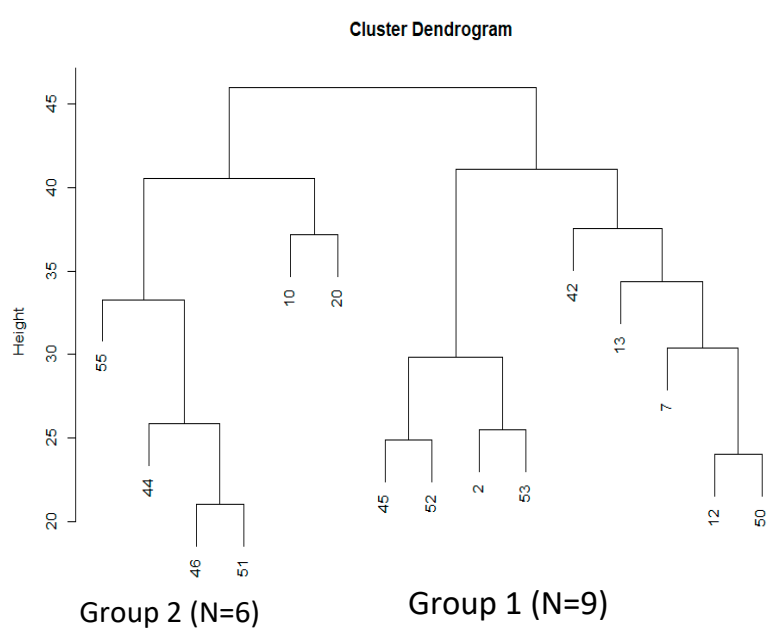

(a)

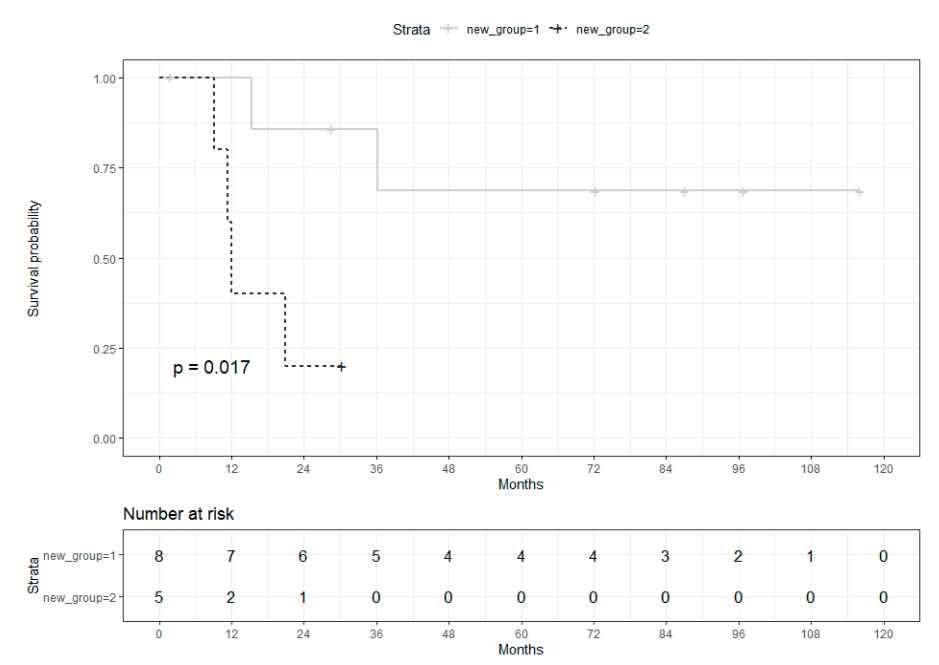

(b)

Figure 2. Subgroup analysis of the TP53mut group only. (a) Unsupervised hierarchical clustering of the TP53mut samples of which mRNA data was available $(n=15)$. (b) Kaplan-Meier curves and log rank test of the overall survival of the two groups identified by hierarchical clustering ( $n=13$, follow-up data of $2 / 15$ cases were not available). 
Table 2. Significant differentially expressed genes between the two clusters within the TP53mut cohort, $p<0.001,+\log 2$ fold change higher gene expression in group 2 vs. group1. - log2fold change: lower gene expression in group 2 vs. group 1.

\begin{tabular}{cccc}
\hline Gene & Log2FC & Base Mean & Gene Name \\
\hline TMPRSS2 & 4.15 & 156.3 & Transmembrane protease serine subtype 2 \\
PLA2G3 & 2.66 & 136.03 & Phospholipase A2 Group III \\
IL20RA & 2.49 & 84.09 & Interleukin 20 Receptor Subunit Alpha \\
RASAL1 & 2.45 & 156.73 & RAS Protein Activator Like 1 \\
IL1R2 & 2.18 & 526.73 & Interleukin 1 Receptor Type 2 \\
PRMT8 & 2.11 & 42.75 & Protein Arginine Methyltransferase 8 \\
MAPK8IP2 & 2.04 & 64.02 & Mitogen-Activated Protein Kinase 8 Interacting Protein 2 \\
PLA2G4A & 1.95 & 127.75 & Phospholipase A2 Group IVA) \\
HES5 & 1.58 & 125.22 & Hes Family BHLH Transcription Factor 5 \\
SYK & 922.43 & Spleen Associated Tyrosine Kinase \\
PTPRR & 1.40 & 55.63 & Protein Tyrosine Phosphatase Receptor Type R \\
TSLP & -3.05 & 78.84 & Thymic stromal lymphopoietin \\
GPC4 & -2.59 & 269.24 & Glypican 4 \\
IGFBP3 & -2.25 & 5798.71 & Prolactin Receptor \\
PRLR & -2.20 & 67.63 & Insulin-like growth factor-binding protein 3 \\
VEGFC & -2.08 & 630.41 & Vascular endothelial growth factor C \\
FN1 & -2.07 & 6497.01 & Fibronectin 1 \\
RET & -1.9 & 75.33 & Rezeptor-Tyrosinkinase \\
DUSP10 & -1.67 & 543.2 & Dual specificity protein phosphatase \\
ITGB3 & -1.25 & 82.5 & (Integrin Subunit Beta 3 \\
\hline
\end{tabular}

The correlation with clinical parameters ( $\mathrm{pT}, \mathrm{pN}$, tumor diameter, invasion depth, grading, recurrence, and survival) revealed a significant difference in overall survival between the two groups, showing a decreased overall survival (OS) in group 2 vs. group $1(p=0.017$, Figure $2 b)$. The median OS of group 2 was 12 months, whereas $>50 \%$ of the patients of group 1 lived longer than the study's follow-up. The correlation of all other clinical parameters (age, recurrence, $\mathrm{pT} / \mathrm{pN}$ status, and tumor size) did not yield any significant results.

\subsubsection{HPV+ VSCC}

In the HPV+ subgroup, the genes involved in cell cycle regulation were particularly often upregulated in comparison to the TP53mut tumors. In the heatmap where HPV+ VSCC cluster together (Figure 1a), the genes involved in G1/S transition, like CDKN2A, CCNE2, CDKN1C, and CDKN2C, as well as S/G2 and G2/M phase regulators CDK2, Wee1, $\mathrm{CDC} 25 \mathrm{C}$, and $\mathrm{CDC7}$, and mini chromosome maintenance complex genes (MCM2/4/5/7) were particularly often upregulated. In addition, genes involved in DNA repair, like BRCA2, BRIP1, FANCA, FANCC, RFC4, PCNA, POLE2, RFC3, and EZH2, were upregulated compared to TP53mut tumors. Other significantly upregulated genes in the HPV+ cluster are shown in Figure 1a,b. None of the HPV+ tumors showed upregulation of cell cycle regulator cyclin D1, which were exclusively upregulated in the TP53mut tumors.

Pathway analysis confirmed the gene expression differences in different cell cycle control pathways (Figure 3; G1/S phase transition, G2/M checkpoints), as well as DNA repair. 


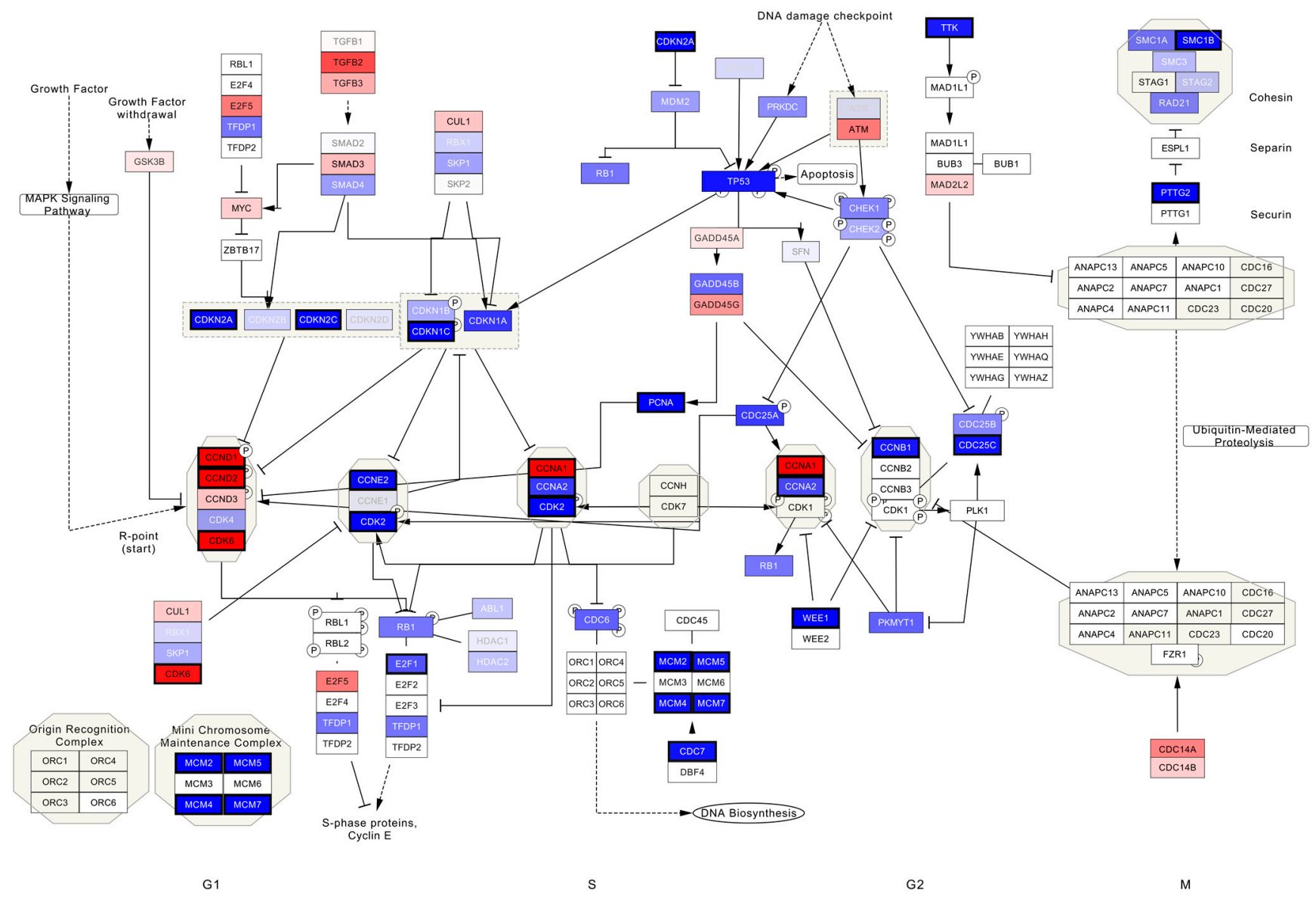

Figure 3. The human cell cycle pathway (WP179). The labels are colored according to the direction of the change in expression. Genes with a higher expression of TP53mut in comparison to HPV+ VSCCs are colored red. A thicker black border marks significantly $(\mathrm{FDR} \leq 0.1)$ differentially expressed genes.

No significant differences with recurrence, $\mathrm{pT} / \mathrm{pN}$ status, tumor size, or survival were found between the HPV+ vs. TP53mut group.

\subsection{3. "OTHER"}

Of the four samples that were grouped as "OTHER", we observed that three $(15,24$, and 56) clustered together in PCA (Figure 4a and Table S4). This small group of three samples showed more similarities in comparison to the TP53mut group than with HPV+ VSCC. Forty-three genes were differentially expressed in the comparison of this group and the HPV+ group, whereas four genes (CDC7, DNMT3A, ITGB8, and SMC1B) were differentially expressed between this and the TP53mut group. Only one gene (DNMT3A) was differentially expressed (downregulated) in comparison to both other subgroups (TP53mut and HPV+ VSCC). 


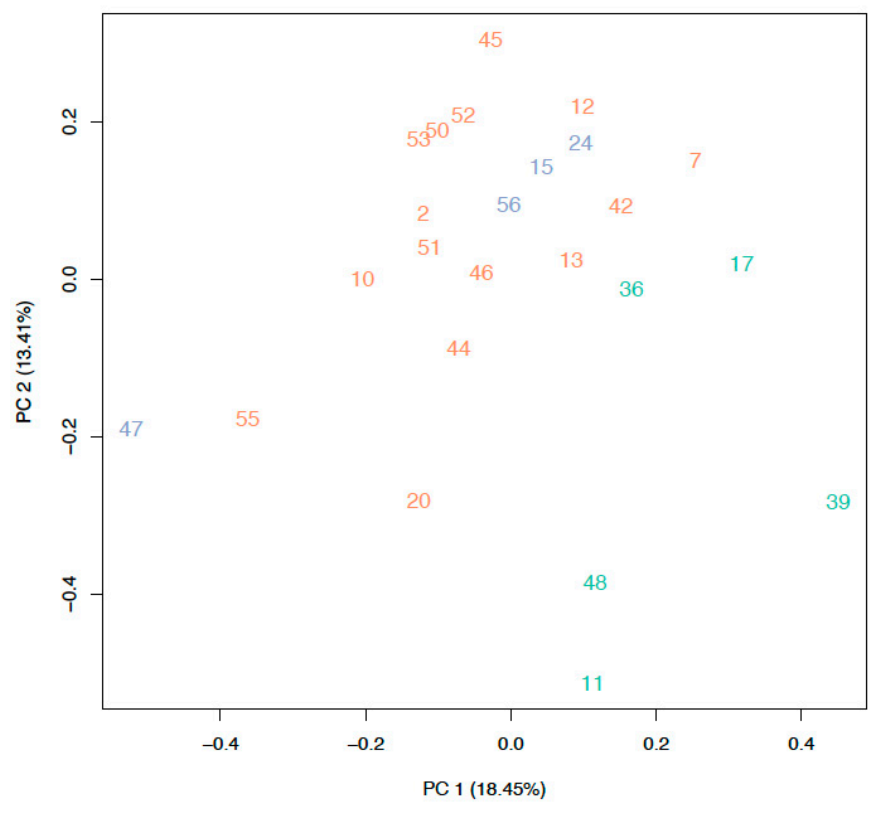

(a)

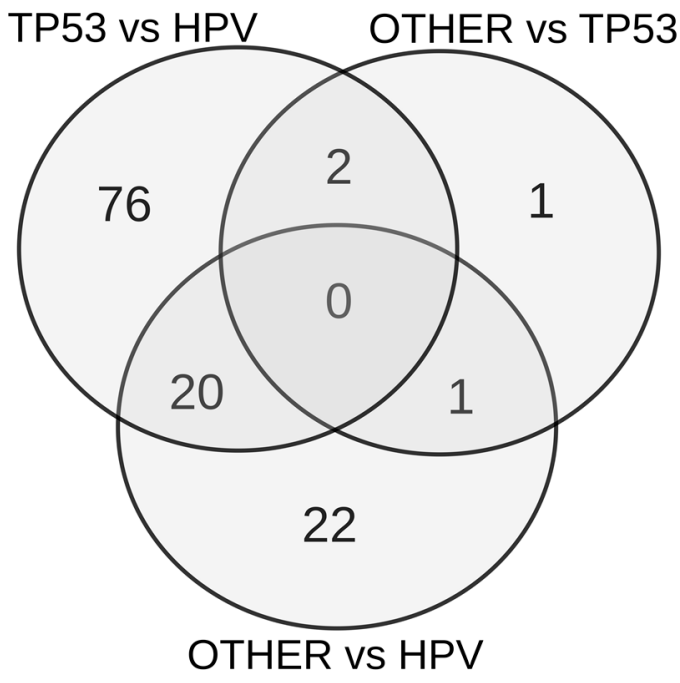

(b)

Figure 4. (a) Principal component analysis (PCA) of the three groups. Red: TP53mut, green: HPV+, blue: “OTHER"; (b) VENN diagram of the differentially expressed genes within the three subgroups; Detailed information on the underlying gene expression data and the intersections shown are provided in the supplements (Table S4).

\section{Discussion}

In the present analysis, the expression profiles of TP53mut VSCC were compared with those of HPV induced VSCC in order to characterize these two subtypes further. The HPV+ cohort showed significantly more differentially expressed genes in cell cycle regulation and DNA repair in the mitotic G1 phase, G1/S phase transition, and G2/M checkpoints. Besides $D K K-1$, which has been linked to a poor prognosis and to cancer progression, as well as displaying the highest log2FC in comparison to HPV+ VSCC, TP53mut tumors showed upregulation of some important key players of cell cycle regulation, e.g., CCND1, CCND2, CCNA1, and CDK6. However, no other commonly dysregulated pathways were identified in TP53mut VSCC. Within the TP53 group, two subgroups with a significant differential expression of 20 genes were identified that correlated with OS.

As expected, a high expression of CDKN2A (p16) was observed as a surrogate for HPV induced transformation in the HPV + cohort. Dysregulation of cell cycle and DNA repair mechanisms has been linked to an overexpression of the viral oncogenes E6/E7. They bind and thus inactivate tumor suppressors $\mathrm{p} 53$ and retinoblastom-protein $(\mathrm{Rb})$, leading to uncontrolled proliferation. Whereas upregulation of cell cycle regulators CDKN2A, $C D K N 1 C, C D K N 2 C, C C N E 2, C D K 2$, Wee1, and $C D C 7$, and mini chromosome maintenance complex genes (MCM2/4/5/7) was detected in the HPV+ cohort, upregulation of critical gatekeepers like CCND1, CCNA1, CCND2, and CDK7 in cell cycle regulation was also observed in the TP53mut group. However, relevance for the tumor suppressor gene CCNA1 has been reported in other HPV induced cancer, e.g., cervical and HNSCC. CCNA1 promoter hypermethylation results in a decreased expression of cyclinA1 mRNA and thereby in a loss of function of the protein [31]. Evidence suggests that E7 of HPV is able to induce CCNA1 promoter methylation by forming a complex with Dnmt1 [32]. In a recently performed genome-wide methylation sequencing on a set of 18 VSCC and 6 normal vulvar tissue, 199 genes were found to be differentially methylated, without CCNA1 being among them [19]. An explanation for this might be the small sample size of HPV induced cancers $(n=3)$ in their cohort. 
CCND1 is considered an oncogene and encodes for the cyclin D1 protein that regulates the transition through the restriction point in the G1 to S phase, thereby promoting proliferation and growth, as well as resistance to chemotherapy and radiotherapy, and a shorter OS $[33,34]$. Recently, several studies have shown that CCND1 amplification is associated with a decreased response to immune checkpoint inhibitors (ICI) [35,36]. In a comprehensive analysis of three large databases (TCGA, Memorial Sloan Kettering Cancer Cancer (MSKCC) databases, and Geneplus cohort), it was revealed that CCND1 amplifications in patients that receive ICIs is associated with decreased OS in different cancer entities. [37]. Furthermore, different cancer hallmarks were associated with CCND1 amplification, including G2M checkpoints, p53 pathway, epithelial-mesenchymal transition, (PI3K)/AKT / mammalian target of rapamycin (mTOR) signaling, KRAS signaling, transforming growth factor (TGF)-b signaling, phosphoinositide 3-kinase, and hypoxia signaling in the pan cancer cohort of the TCGA. Interestingly, HNSCC that share distinct biological characteristics with vulvar cancer show a particularly high occurrence of CCND1 amplifications (31\%) in the TP53mut subgroup [20]. Other frequently amplified genes in the TCGA head and neck cohort, like EGFR, FGFR1, and MYC, were analyzed in our panel, but were not differentially regulated in TP53mut vs. HPV+ VSCC. The gene expression analysis from the TCGA database showed that CCND1 amplification was significantly related to the upregulation of the mRNA expression of CCND1 across the top nine cancer types. In our previous WES copy number analysis, significant differences were found in gain of chromosome 11, 67,000,001-71,000,001 bp between the TP53mut and HPV+ group [5]. This gain along chromosome 11 includes CCND1, and might be responsible for the gene's overexpression. Cyclin D1 mutations are generally a rare event-only one somatic mutation was detected in our WES cohort. In a recently published targeted NGS analysis of 280 VSCC samples in 406 genes, Williams et al. demonstrated a high CCND1 amplification rate in HPV- vs. HPV+ VSCC $(22 \%$ vs. $2 \% ; p<0.001)$ [17]. In vulvar cancer, $C C N D 1$ overexpression has been shown to be significantly related to the presence of regional lymph node metastases $(p<0.001)$ and HPV negativity $(p<0.001)$ [38].

In a recent RNA sequencing analysis published by Kolitz et al., VSCC were analyzed for differential expressed genes in HPV+ vs. HPV - tumors [23]. E2F was upregulated in HPV+ cases, following HPV E7 activation, and EYA2 was also upregulated, which has been shown to promote growth and migration in cervical cancer. In comparison to our own work, no overlapping expression patterns were detected. Differences in methodology, e.g., panel vs. whole transcriptome, subtype stratification (WES vs. polymerase chain reaction (PCR) and in situ hybridization (ISH)), and fresh frozen vs. paraffin embedded tissue preparation limits comparability.

Most of the differentially expressed genes reported by Kolitz et al. were not detected in our data and vice versa. However, STMN1 (Stathmin1) was also upregulated in HPV+ vs. TP53mut (log2FC: - 1.04; base mean: 3254) VSCC in our analysis. Stathmin1 has previously been reported to be a highly sensitive and specific biomarker for the diagnosis of vulvar high-grade squamous epithelial lesion (HSIL) by Nooij et al. [39].

Activation of the PIK3/Akt/mTOR pathway by somatic mutation, PIK3CA copy number gains, or indirect regulatory effects such as the abrogation of cellular $m T O R$ degradation via a loss of function of $F B X W 7$ has been reported across all NGS studies. It has predominantly been reported for HPV+ VSCC, but also in HPV- VSCC. Our expression data show a downregulation of PIK3R1 and PIK3R3 expression in TP53mut vs. HPV+ VSCC, encoding the regulatory isoforms p $85 \alpha$ and p55 $\gamma$ of PI3K. Accumulating evidence also suggests that changes in p85 $\alpha$ levels can modulate PI3K activation. It was reported that $\mathrm{p} 85 \alpha$ depletion increases PI3K/AKT signaling and transformation in vitro, and accelerates tumor development [40]. A differential expression of FBXW7 was not observed in our analysis, however NOTCH1, one of its main targets, which it targets for degradation, was differentially expressed in our dataset. In 2017, a third HPV independent/TP53wt pathogenesis was first suggested by Nooij et al., later termed as "double negative" [41]. In a small cohort of 19 VSCC subjected to targeted NGS panel analysis, a high number of NOTCH1 mutations were 
observed in HPV - / TP53wt subgroup (5/10). This subtype was not subject of our analysis, however, as we focused on differential expression between the so far clearly identified two prognostically relevant subtypes of VSCC. However, upregulation of NOTCH1 was also observed in HPV+ vs. TP53mut VSCC in our cohort. NOTCH1 is a transmembrane receptor involved in different cancer hallmarks like differentiation, proliferation, metastasis, apoptosis, and chemoresistance [42]. A variety of NOTCH inhibitors are being investigated in clinical trials, with a first phase III trial of inhibitor of the oral Nirogacesta, a $\gamma$-secretase inhibitor currently recruiting (NCT03785964). Our data add to the hypothesis of molecular heterogeneity among HPV independent VSCC. We observed that three of the four samples that were neither related to TP53mut nor HPV+ group show several similarities among them. In PCA, they are enclosed in a TP53mut subgroup and indeed show more similarities with TP53mut VSCC in terms of the gene expression pattern. Only DNMT3A was differentially expressed (downregulated) to TP53mut and HPV+ VSCC. DNMT3A belongs to a family of de novo DNA methyltransferases [43] and has traditionally been considered an oncogene, but can also act as a tumor suppressor under certain circumstances [44]. Just recently, hypermethylation of $D N M T 3 A$ was reported in the above referenced genome-wide methylation sequencing in VSCC. Previously, an overexpression of DNMT3A was reported to be associated with increased risk of local recurrence in VSCC $(\mathrm{HR}=4.5, p=0.012)$, also in multivariate analysis after adjustment for disease stage $(\mathrm{HR}=6.00, p=0.003)$ and groin node metastasis ( $\mathrm{HR}=4.81, p=0.008)$. Correlation with $\mathrm{p} 16$ staining revealed that the overexpression of DNMT3A was significantly correlated to $\mathrm{p} 16$ negativity. Whether there is clinical relevance in this TP53wt/HPV - vulvar cancer subgroup can only be speculated upon at this point and will need further validation.

In TP53mut VSCC, the highest expression of DKK-1 was observed compared to HPV+ VSCC in our cohort. DKK-1 was originally described as a tumor suppressor by blocking the b-catenin dependent Wnt signaling pathway, which is frequently overactivated in cancer. More recently, elevated DKK-1 serum levels and protein expression were observed in a variety of tumor entities (e.g., gastric, breast, ovarian, and pancreatic cancer). Therefore, gene expression profiles were analyzed in the large TCGA cohort of 9677 tumor samples and 916 normal samples [45]. It was shown that DKK-1 mRNA was overexpressed in a wide range of tumors, e.g., head and neck, and pancreas, and was correlated with a shorter DFS. Early clinical study combinations of antiDKK-1 Ab may improve the response to $\mathrm{PD} / \mathrm{PD}-\mathrm{L} 1$ therapy via the reduction of myeloid derived suppressor cells and upregulation in $\mathrm{CD} 45+$.

This analysis indicates that there might be evidence for a further subgroup within the TP53mut group that needs to be characterized in the future. Within the TP53mut group, two subgroups were identified with distinct mRNA expression patterns that correlated with survival. Nevertheless, the results should be interpreted with caution as the subgroups were very small in this cohort. The results should be confirmed in a larger independent cohort. The highest expression of TMPRSS2, RASAL1, PLA2G3, HES5, IL1R2, and PLA2G4A was shown in cluster 2 vs. cluster 1, and a lower expression was observed in IGFBP3, $F N 1, V E G F C$, and GPC4 in cluster 2 vs. cluster 1, respectively. To our knowledge, none of these genes have been linked to vulvar cancer pathogenesis so far. However, for some of the genes, clinical relevance has been shown in other tumor entities, e.g., in prostate cancer, an overexpression of TMPRSS2 has been correlated to cancer cell invasion and metastasis [46], and a high IL1R2 expression has been linked to a decreased overall survival and relapse-free survival in breast cancer patients [47]. HES5 is a key regulator of NOTCH signaling and displays context-dependent oncogenic and tumor suppressive features in liver carcinogenesis [48].

Validation of these results in a larger second independent cohort including a subset of normal tissue will be the next step to confirm our results. As this analysis is focused on the differences between TP53mut vs. HPV+ VSCC, the results of the present expression analysis can only highlight the differentially expressed genes between the two subgroups. 
Expression changes that are relevant in both pathogenic pathways, like the PIK3/Akt/mTOR pathway, might therefore, not be identified.

\section{Conclusions}

RNA expression profiles show a distinct regulation of selective candidate genes with regard to the known VSCC subtypes and potentially enable further subclassification in the TP53mut group. For HPV+ VSCC, upregulation of the cell cycle regulators was predominantly seen. A small subset of three HPV-/TP53wt VSCC were classified as "other", but were shown to share most RNA expression patterns with TP53mut rather than $\mathrm{HPV}+\mathrm{VSCC}$.

Supplementary Materials: The following are available online at https://www.mdpi.com/article/ $10.3390 /$ cancers $13246372 / s 1$. Table S1 Somatic variants from whole exome sequencing analysis in vulvar cancer. Table S2: Results of the differential expression analysis (TP53mut vs. HPV+). Table S3: Individual patient characteristics of TP53mutated, HPV positive and "double" negative vulvar cancer. Table S4: Results of the differential expression analysis ("OTHER" vs. TP53mut and "OTHER" vs. $\mathrm{HPV+}$ ) and intersections between all contrasts.

Author Contributions: All of the authors made substantial intellectual contributions to the article, provided critical feedback, and gave approval of the final manuscript to be published. K.P. initial design, conception and coordination of the study, analyzed and interpreted the data, and drafted the manuscript. M.A., bioinformatic and statistical analysis, and helped to draft the manuscript. L.O.-F. and K.E., participated in the study design, supervision of laboratory work, technical support, and revised the manuscript critically. P.L., N.C.B. and E.B. pathological studies and revised the manuscript critically. S.R., B.S., A.J. and M.C.W. supervised the project by providing substantial feedback and revising it critically for intellectual content. S.A.J. conception and coordination of the study, statistically analyzed and interpreted the data, and helped to draft the manuscript. L.W. initial design, conception, and coordination of the study; analyzed and interpreted the data; and helped to draft the manuscript. All authors have read and agreed to the published version of the manuscript.

Funding: This research was funded by Hamburger Krebsgesellschaft (project number: 4016). K.P. and S.A.J. are scholars of the Deutsche Krebshilfe and receive funding within the Mildred Scheel Cancer Career Center, HaTriCS4 program at the University Medical Center Hamburg-Eppendorf, Hamburg, Germany.

Institutional Review Board Statement: The study was conducted according to the guidelines of the Declaration of Helsinki and was approved by the Institutional Review Board and Ethics Committee of the Medical Board Hamburg (reference number 190504).

Informed Consent Statement: Informed consent was obtained from all subjects involved in the study.

Data Availability Statement: The data presented in this study are available in supplementary material.

Conflicts of Interest: K.P. reports personal fees from AstraZeneca, personal fees from MSK, personal fees from Molecular health, personal fees from Gsk, personal fees from Roche, personal fees from Clovis Oncology, outside the submitted work. A.J. reports personal fees from Astra Zeneca, personal fees from Molecular Health, personal fees from Gsk, personal fees from Roche, personal fees from Clovis Oncology, personal fees from MSD, outside the submitted work. L.W. reports personal fees from med update $\mathrm{GmbH}$, grants, personal fees and non-financial support from medac oncology, personal fees from promedics $\mathrm{GmbH}$, grants, personal fees and non-financial support from Roche, grants, personal fees and non-financial support from Tesaro, personal fees from Teva, personal fees from OmniaMed, personal fees from Pfizer, personal fees from Greiner, outside the submitted work. All of the other authors declare no conflict of interest.

\section{References}

1. Buttmann-Schweiger, N.; Klug, S.J.; Luyten, A.; Holleczek, B.; Heitz, F.; Du Bois, A.; Kraywinkel, K. Incidence Patterns and Temporal Trends of Invasive Nonmelanotic Vulvar Tumors in Germany 1999-2011. A Population-Based Cancer Registry Analysis. PLoS ONE 2015, 10, e0128073. [CrossRef]

2. Holleczek, B.; Sehouli, J.; Barinoff, J. Vulvar cancer in Germany: Increase in incidence and change in tumour biological characteristics from 1974 to 2013. Acta Oncol. 2018, 57, 324-330. [CrossRef] [PubMed] 
3. De Martel, C.; Georges, D.; Bray, F.; Ferlay, J.; Clifford, G.M. Global burden of cancer attributable to infections in 2018: A worldwide incidence analysis. Lancet Glob. Health 2020, 8, e180-e190. [CrossRef]

4. Woelber, L.; Jaeger, A.; Prieske, K. New treatment standards for vulvar cancer. Curr. Opin. Obstet. Gynecol. 2020, 32, 9-14. [CrossRef]

5. Prieske, K.; Alawi, M.; Oliveira-Ferrer, L.; Jaeger, A.; Eylmann, K.; Burandt, E.; Schmalfeldt, B.; Joosse, S.A.; Woelber, L. Genomic characterization of vulvar squamous cell carcinoma. Gynecol. Oncol. 2020, 158, 547-554. [CrossRef] [PubMed]

6. Schuurman, M.; Einden, L.V.D.; Massuger, L.; Kiemeney, L.; van der Aa, M.; de Hullu, J. Trends in incidence and survival of Dutch women with vulvar squamous cell carcinoma. Eur. J. Cancer 2013, 49, 3872-3880. [CrossRef]

7. Woelber, L.; Prieske, K.; Eulenburg, C.; Oliveira-Ferrer, L.; de Gregorio, N.; Klapdor, R.; Kalder, M.; Braicu, I.; Fuerst, S.; Klar, M.; et al. p53 and p16 expression profiles in vulvar cancer-A translational analysis by the AGO-CaRE- study group. Am. J. Obstet. Gynecol. 2021, 224, 595.e1-595.e11. [CrossRef] [PubMed]

8. Nooij, L.S.; Ter Haar, N.T.; Ruano, D.; Rakislova, N.; Van Wezel, T.; Smit, V.T.; Trimbos, B.J.; Ordi, J.; Van Poelgeest, M.I.; Bosse, T. Genomic Characterization of Vulvar (Pre)cancers Identifies Distinct Molecular Subtypes with Prognostic Significance. Clin. Cancer Res. 2017, 23, 6781-6789. [CrossRef]

9. Mahner, S.; Jueckstock, J.; Hilpert, F.; Neuser, P.; Harter, P.; de Gregorio, N.; Hasenburg, A.; Sehouli, J.; Habermann, A.; Hillemanns, P.; et al. Adjuvant Therapy in Lymph Node-Positive Vulvar Cancer: The AGO-CaRE-1 Study. J. Natl. Cancer Inst. 2015, 107, dju426. [CrossRef] [PubMed]

10. Prieske, K.; Haeringer, N.; Grimm, D.; Trillsch, F.; Eulenburg, C.; Burandt, E.; Schmalfeldt, B.; Mahner, S.; Mueller, V.; Woelber, L. Patterns of distant metastases in vulvar cancer. Gynecol. Oncol. 2016, 142, 427-434. [CrossRef]

11. Chung, H.; Ros, W.; Delord, J.-P.; Perets, R.; Italiano, A.; Shapira-Frommer, R.; Manzuk, L.; Piha-Paul, S.; Xu, L.; Zeigenfuss, S.; et al. Efficacy and Safety of Pembrolizumab in Previously Treated Advanced Cervical Cancer: Results From the Phase II KEYNOTE-158 Study. J. Clin. Oncol. 2019, 37, 1470-1478. [CrossRef]

12. Tewari, K.S.; Sill, M.W.; Long, H.J., 3rd; Penson, R.T.; Huang, H.; Ramondetta, L.M.; Landrum, L.M.; Oaknin, A.; Reid, T.J.; Leitao, M.M.; et al. Improved Survival with Bevacizumab in Advanced Cervical Cancer. N. Engl. J. Med. 2014, 370, 734-743. [CrossRef] [PubMed]

13. Sabatini, M.E.; Chiocca, S. Human papillomavirus as a driver of head and neck cancers. Br. J. Cancer 2019, 122, 306-314. [CrossRef]

14. Trietsch, M.D.; Nooij, L.S.; Gaarenstroom, K.; van Poelgeest, M.I. Genetic and epigenetic changes in vulvar squamous cell carcinoma and its precursor lesions: A review of the current literature. Gynecol. Oncol. 2015, 136, 143-157. [CrossRef]

15. Zięba, S.; Kowalik, A.; Zalewski, K.; Rusetska, N.; Goryca, K.; Piaścik, A.; Misiek, M.; Bakuła-Zalewska, E.; Kopczyński, J.; Kowalski, K.; et al. Somatic mutation profiling of vulvar cancer: Exploring therapeutic targets. Gynecol. Oncol. 2018, 150, 552-561. [CrossRef]

16. Weberpals, J.I.; Lo, B.; Duciaume, M.M.; Spaans, J.N.; Clancy, A.; Dimitroulakos, J.; Goss, G.D.; Sekhon, H.S. Vulvar Squamous Cell Carcinoma (VSCC) as Two Diseases: HPV Status Identifies Distinct Mutational Profiles Including Oncogenic Fibroblast Growth Factor Receptor. Clin. Cancer Res. 2017, 23, 4501-4510. [CrossRef] [PubMed]

17. Williams, E.A.; Werth, A.J.; Sharaf, R.; Montesion, M.; Sokol, E.S.; Pavlick, D.C.; McLaughlin-Drubin, M.; Erlich, R.; Toma, H.; Williams, K.J.; et al. Vulvar Squamous Cell Carcinoma: Comprehensive Genomic Profiling of HPV+ Versus HPV- Forms Reveals Distinct Sets of Potentially Actionable Molecular Targets. JCO Precis. Oncol. 2020, 4, 647-661. [CrossRef]

18. Han, M.-R.; Shin, S.; Park, H.-C.; Kim, M.S.; Lee, S.H.; Jung, S.H.; Song, S.Y.; Lee, S.H.; Chung, Y.-J. Mutational signatures and chromosome alteration profiles of squamous cell carcinomas of the vulva. Exp. Mol. Med. 2018, 50, e442. [CrossRef]

19. Dasgupta, S.; Ewing-Graham, P.; Swagemakers, S.; Bosch, T.V.D.; Atmodimedjo, P.; Verbiest, M.; de Haan, M.; van Doorn, H.; van der Spek, P.; Koljenović, S.; et al. Exploring Differentially Methylated Genes in Vulvar Squamous Cell Carcinoma. Cancers 2021, 13, 3580. [CrossRef] [PubMed]

20. Cancer Genome Atlas Network. Comprehensive genomic characterization of head and neck squamous cell carcinomas. Nature 2015, 517, 576-582. [CrossRef] [PubMed]

21. The Cancer Genome Atlas Research Network. Integrated genomic and molecular characterization of cervical cancer. Nature 2017, 543, 378-384. [CrossRef]

22. Pappa, K.I.; Jacob-Hirsch, J.; Vlachos, G.D.; Christodoulou, I.; Partsinevelos, G.; Amariglio, N.; Markaki, S.; Antsaklis, A.; Anagnou, N.P. Expression Profiling of Vulvar Carcinoma: Clues for Deranged Extracellular Matrix Remodeling and Effects on Multiple Signaling Pathways Combined with Discrete Patient Subsets. Transl. Oncol. 2011, 4, 301-IN6. [CrossRef]

23. Kolitz, E.; Lucas, E.; Hosler, G.A.; Kim, J.; Hammer, S.; Lewis, C.; Xu, L.; Day, A.T.; Mauskar, M.; Lea, J.S.; et al. Human Papillomavirus-Positive and -Negative Vulvar Squamous Cell Carcinoma Are Biologically but Not Clinically Distinct. J. Investig. Dermatol. 2021. [CrossRef]

24. Beller, U.; Quinn, M.A.; Benedet, J.L.; Creasman, W.T.; Ngan, H.Y.S.; Maisonneuve, P.; Pecorelli, S.; Odicino, F.; Heintz, A. Carcinoma of the Vulva. Int. J. Gynecol. Obstet. 2006, 95, S7-S27. [CrossRef]

25. Benedet, J.L.; Bender, H.; Jones, H., 3rd; Ngan, H.Y.; Pecorelli, S. FIGO staging classifications and clinical practice guidelines in the management of gynecologic cancers. FIGO Committee on Gynecologic Oncology. Int. J. Gynaecol. Obstet. $2000,70,209-262$.

26. Risso, D.; Ngai, J.; Speed, T.P.; Dudoit, S. Normalization of RNA-seq data using factor analysis of control genes or samples. Nat. Biotechnol. 2014, 32, 896-902. [CrossRef] 
27. Bhattacharya, A.; Hamilton, A.M.; Furberg, H.; Pietzak, E.; Purdue, M.P.; Troester, M.A.; Hoadley, K.A.; Love, M.I. An approach for normalization and quality control for NanoString RNA expression data. Brief. Bioinform. 2021, 22, bbaa163. [CrossRef] [PubMed]

28. Wu, T.; Hu, E.; Xu, S.; Chen, M.; Guo, P.; Dai, Z.; Feng, T.; Zhou, L.; Tang, W.; Zhan, L.; et al. clusterProfiler 4.0: A universal enrichment tool for interpreting omics data. Innovation 2021, 2, 100141. [CrossRef]

29. Lein, E.S.; Hawrylycz, M.J.; Ao, N.; Ayres, M.; Bensinger, A.; Bernard, A.; Boe, A.F.; Boguski, M.S.; Brockway, K.S.; Byrnes, E.J.; et al. Genome-wide atlas of gene expression in the adult mouse brain. Nature 2007, 445, 168-176. [CrossRef] [PubMed]

30. Joosse, S.A. In-Silico Online (Version 2.1.2). 2019. Available online: http://in-silico.online (accessed on 16 March 2021).

31. Weiss, D.; Basel, T.; Sachse, F.; Braeuninger, A.; Rudack, C. Promoter methylation of cyclin A1 is associated with human papillomavirus 16 induced head and neck squamous cell carcinoma independently of p53 mutation. Mol. Carcinog. 2011, 50, 680-688. [CrossRef] [PubMed]

32. Chalertpet, K.; Pakdeechaidan, W.; Patel, V.; Mutirangura, A.; Yanatatsaneejit, P. Human papillomavirus type 16 E7 oncoprotein mediates CCNA1 promoter methylation. Cancer Sci. 2015, 106, 1333-1340. [CrossRef]

33. Malumbres, M.; Barbacid, M. To cycle or not to cycle: A critical decision in cancer. Nat. Rev. Cancer 2001, 1, 222-231. [CrossRef] [PubMed]

34. Musgrove, E.A.; Caldon, C.E.; Barraclough, J.; Stone, A.; Sutherland, R.L. Cyclin D as a therapeutic target in cancer. Nat. Rev. Cancer 2011, 11, 558-572. [CrossRef] [PubMed]

35. Saâda-Bouzid, E.; Defaucheux, C.; Karabajakian, A.; Coloma, V.P.; Servois, V.; Paoletti, X.; Even, C.; Fayette, J.; Guigay, J.; Loirat, D.; et al. Hyperprogression during anti-PD-1/PD-L1 therapy in patients with recurrent and/or metastatic head and neck squamous cell carcinoma. Ann. Oncol. 2017, 28, 1605-1611. [CrossRef]

36. Yu, J.; Yan, J.; Guo, Q.; Chi, Z.; Tang, B.; Zheng, B.; Yu, J.; Yin, T.; Cheng, Z.; Wu, X.; et al. Genetic Aberrations in the CDK4 Pathway Are Associated with Innate Resistance to PD-1 Blockade in Chinese Patients with Non-Cutaneous Melanoma. Clin. Cancer Res. 2019, 25, 6511-6523. [CrossRef] [PubMed]

37. Chen, Y.; Huang, Y.; Gao, X.; Li, Y.; Lin, J.; Chen, L.; Chang, L.; Chen, G.; Guan, Y.; Pan, L.K.; et al. CCND1 Amplification Contributes to Immunosuppression and Is Associated With a Poor Prognosis to Immune Checkpoint Inhibitors in Solid Tumors. Front. Immunol. 2020, 11, 1620. [CrossRef]

38. Choschzick, M.; Hess, S.; Tennstedt, P.; Holst, F.; Bohlken, H.; Gieseking, F.; Mahner, S.; Woelber, L.; Simon, R.; Sauter, G. Role of cyclin D1 amplification and expression in vulvar carcinomas. Hum. Pathol. 2012, 43, 1386-1393. [CrossRef] [PubMed]

39. Nooij, L.S.; Dreef, E.J.; Smit, V.T.; van Poelgeest, M.I.; Bosse, T. Stathmin is a highly sensitive and specific biomarker for vulvar high-grade squamous intraepithelial lesions. J. Clin. Pathol. 2016, 69, 1070-1075. [CrossRef]

40. Thorpe, L.M.; Spangle, J.M.; Ohlson, C.E.; Cheng, H.; Roberts, T.M.; Cantley, L.C.; Zhao, J.J. PI3K-p110 $\alpha$ mediates the oncogenic activity induced by loss of the novel tumor suppressor PI3K-p85 $\alpha$. Proc. Natl. Acad. Sci. USA 2017, 114, 7095-7100. [CrossRef] [PubMed]

41. Zeimet, A.G. Molecular characterization of vulvar squamous cell cancer: High time to gain ground. Gynecol. Oncol. 2020, 158, 519-520. [CrossRef] [PubMed]

42. Moore, G.; Annett, S.; McClements, L.; Robson, T. Top Notch Targeting Strategies in Cancer: A Detailed Overview of Recent Insights and Current Perspectives. Cells 2020, 9, 1503. [CrossRef]

43. Leonard, S.; Pereira, M.; Fox, R.; Gordon, N.; Yap, J.; Kehoe, S.; Luesley, D.; Woodman, C.; Ganesan, R. Over-expression of DNMT3A predicts the risk of recurrent vulvar squamous cell carcinomas. Gynecol. Oncol. 2016, 143, 414-420. [CrossRef]

44. Chen, B.-F.; Chan, W.-Y. The de novo DNA methyltransferase DNMT3A in development and cancer. Epigenetics 2014, 9, 669-677. [CrossRef] [PubMed]

45. Chu, H.Y.; Chen, Z.; Wang, L.; Zhang, Z.-K.; Tan, X.; Liu, S.; Zhang, B.-T.; Lu, A.; Yu, Y.; Zhang, G. Dickkopf-1: A Promising Target for Cancer Immunotherapy. Front. Immunol. 2021, 12, 658097. [CrossRef] [PubMed]

46. Ko, C.-J.; Hsu, T.-W.; Wu, S.-R.; Lan, S.-W.; Hsiao, T.-F.; Lin, H.-Y.; Tu, H.-F.; Lee, C.-F.; Huang, C.-C.; Chen, M.-J.M.; et al. Inhibition of TMPRSS2 by HAI-2 reduces prostate cancer cell invasion and metastasis. Oncogene 2020, 39, 5950-5963. [CrossRef]

47. Zhang, L.; Qiang, J.; Yang, X.; Wang, D.; Rehman, A.U.; He, X.; Chen, W.; Sheng, D.; Zhou, L.; Jiang, Y.; et al. IL1R2 Blockade Suppresses Breast Tumorigenesis and Progression by Impairing USP15-Dependent BMI1 Stability. Adv. Sci. 2019, 7, 1901728. [CrossRef] [PubMed]

48. Luiken, S.; Fraas, A.; Bieg, M.; Sugiyanto, R.; Goeppert, B.; Singer, S.; Ploeger, C.; Warsow, G.; Marquardt, J.U.; Sticht, C.; et al. NOTCH target gene HES5 mediates oncogenic and tumor suppressive functions in hepatocarcinogenesis. Oncogene 2020, 39, 3128-3144. [CrossRef] [PubMed] 